\title{
NOTES
}

\section{An effective, cheap, and relatively nontraumatizing rat restrainer}

\section{W. P. BELLINGHAM}

Australian National University, Canberra, A. C. T., Australia

For the past several years we have used a simple but effective procedure for comfortably restraining rats over long periods of time, which may be of interest. The "rat bag" consists of a cloth funnel with Velcro tabs sewn along the narrow side of the funnel (see Figure 1). The size of the funnel can be varied to suit the rat. The large end is held open, and the rat is encouraged to enter. Once the animal is inside, the end of the bag is closed around the base of the tail. The rat and bag are then held much like an ice cream cone, with the rat's nose pointed toward the ceiling. This results in the rat's climbing tightly into the bag, whereupon the Velcro tab is firmly closed such that the narrow end of the bag is sealed behind the ears, across the neck, and in front of the shoulders of the animal. Then a freezer bag tie or piece of string is wrapped around the bag at the base of the tail, but not tightly enough to cut off circulation.

The result is that the rat is firmly but comfortably held, unable to move forward because of the narrowing of the funnel and unable to move backward because of the tie. It is important that the diameter and angle of the funnel be such that the rat is firmly surrounded by the cloth in order to minimize movement and prevent the rat from rotating inside the bag. A few practice sizes and shapes should result in a proper fit.

This technique has innumerable advantages. The head and tail are exposed. We, for instance, routinely use the rat bag for intravenous tail injections in work on taste aversions. The bag and a hammock are used in order to expose rats to long series of light and tone stimuli in habituation studies. Both needle and paste-on electrodes are placed inside the bag to record muscular movement correlated with stimulus onset. In several cases rats have been confined via rat bags in the apparatus for up to $8 \mathrm{~h}$. The bag appears to significantly reduce trauma. Rats left in the bag in a quiet environment for even short periods of time fall asleep. In any case, the soft cloth enclosure and gentle but even pressure produce considerably less struggling than any of the many techniques previously tried.

The technique is quite useful for intraperitoneal and intramuscular injections, especially for the novice. The bag also significantly facilitates recovery from surgery. Rats with implanted electrodes have been placed postoperatively into the bags in order to keep the animals

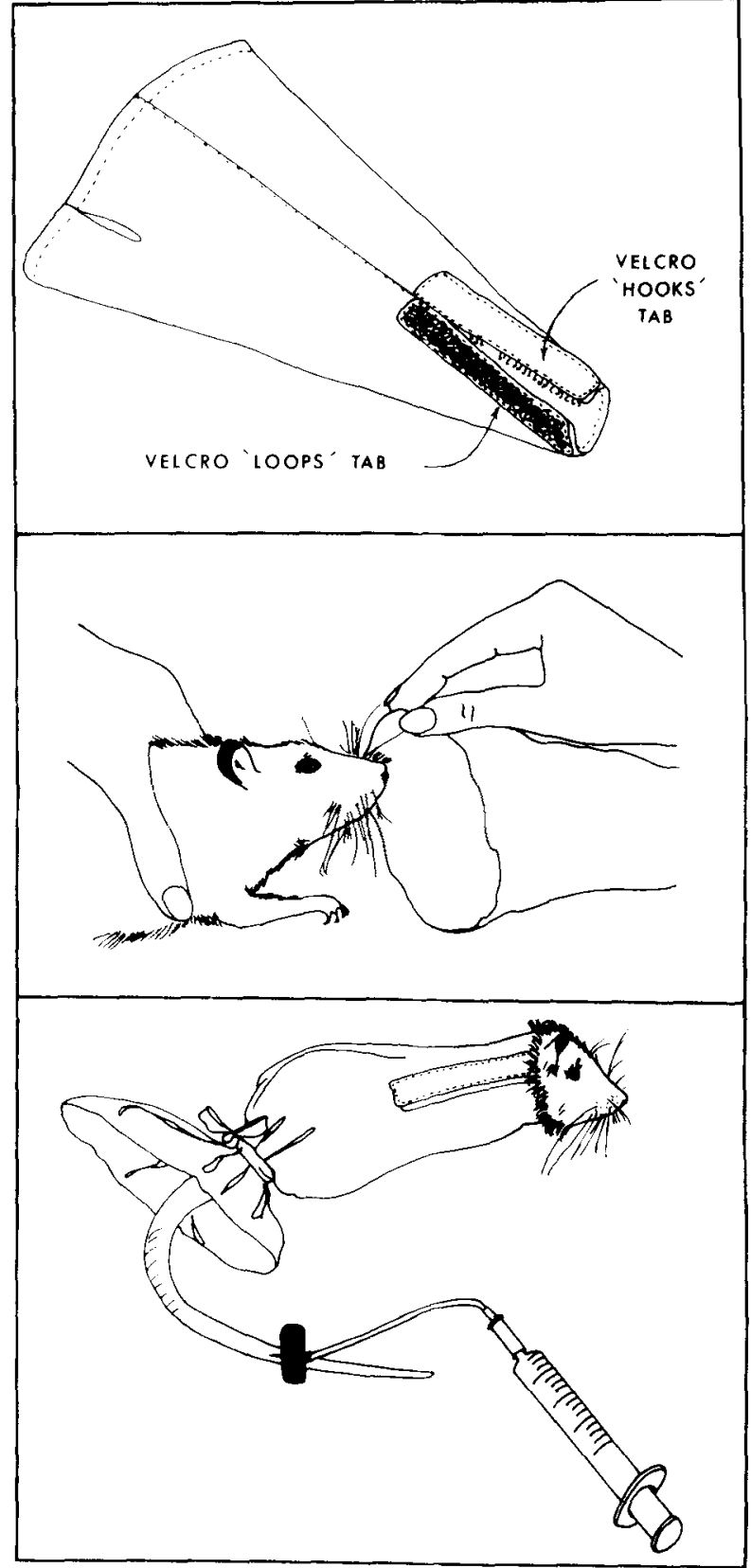

Figure 1. An illustration of the bag and a rat being placed in the bag and secured for intravenous injection.

quiet and warm. It also prevents the rat from attacking the stitches and electrode mount.

If bottles are mounted and food is contained appropriately, it is easy to train the rat to eat and drink while confined. The bags are also easy to clean. 
This remarkably useful and simple device was first reported by Shadle and Skarupinski (1935); various versions of it have undoubtedly been used since then. However, many present-day researchers are unfamiliar with the device, and a more contemporary reiteration seems needed. The only modification is to substitute Velcro for the Shadle and Skarupinski zipper. Occasionally, an animal's fur is jammed with a zipper and/or the rat is pinched. When experimental purposes dictate the need for confinement, this technique is highly recommended as most humane.

\section{REFERENCE}

Shadle, A. R., \& Skarupinski, I. A zipper tube for holding small live animals. Science, 1935, 82, 335.

(Accepted for publication January 15, 1980.) 